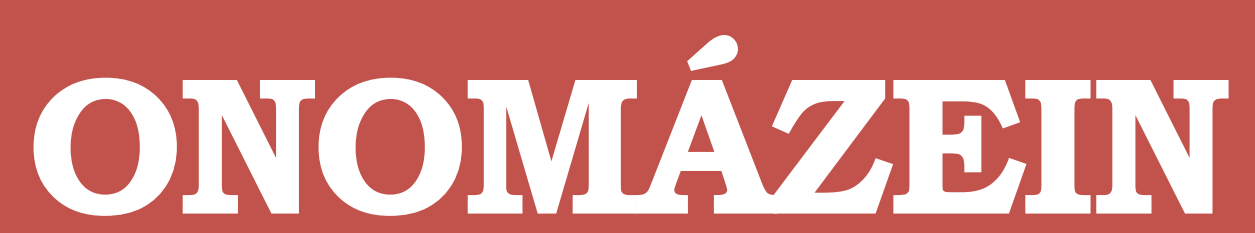

Revista de lingüística, filología y traducción
PONTIFICIA UNIVERSIDAD CATÓLICA DE CHILE FACULTAD DE LETRAS

\title{
El futuro perfecto y los predicados estativos ${ }^{1}$
}

The future perfect and the stative predicates

\section{María Martínez-Atienza}

Universidad de Córdoba

España

\section{(c) $($ i $)$}

María Martínez-Atienza: Departamento de Ciencias del Lenguaje, Facultad de Filosofía y Letras, Universidad de Córdoba, España. | E-mail:mmartinezatienza@uco.es 


\section{Resumen}

En este trabajo analizamos el significado aspectual que recibe el futuro compuesto cuando se combina con predicados estativos desde el punto de vista del aspecto léxico o modo de acción. Como demostramos, la variedad expresada en estos casos es el denominado perfecto experiencial, de acuerdo con la cual se expresa la experiencia del sujeto en el momento de la enunciación o en un determinado punto de referencia como resultado de un evento que ha tenido lugar al menos en una ocasión. Estudiaremos para ello las dos interpretaciones que recibe la forma verbal objeto de nuestro estudio: de anterioridad respecto a un evento posterior al momento de la enunciación (con carácter relativo) y de modalidad epistémica (con carácter absoluto).

Palabras clave: futuro compuesto; significado aspectual; predicados estativos; subvariedades de perfecto; perfecto experiencial.

\section{Abstract}

In this paper we analyze the aspectual meaning that the future perfect receives when it is combined with stative predicates from the point of view of the lexical aspect or mode of action. As we show, the variety expressed in these cases is the so-called experiential perfect, according to which the experience of the subject is expressed at the enunciation moment or at a certain point of reference as a result of an event that has taken place at least on one occasion. With this objective, we will study the two interpretations that this verbal form receives: of anteriority with respect to an event subsequent to the moment of the enunciation (with relative character) and of epistemic modality (with absolute character).

Keywords: future perfect; aspectual meaning; stative predicates; subvarieties of perfect; experiential perfect.

1 Este trabajo se inscribe en el marco del proyecto HISPANAGRAMA "Las ideas gramaticales en la América del Pacífico y El Caribe (1800-1950: fuentes, focos, series textuales y canon)" [FFI201786335-P] del MINECO, Plan Estatal de Investigación Científica y Técnica y de Innovación, de cuyo equipo investigador la autora del artículo forma parte y cuyos IP son Alfonso Zamorano Aguilar (IP1) y Esteban T. Montoro del Arco (IP2). 


\section{Introducción e hipótesis de trabajo}

El futuro compuesto, formado por el auxiliar haber en futuro más el participio del verbo que se conjugue, puede recibir dos interpretaciones:

1) Interpretación de anterioridad respecto a un futuro, de acuerdo con la cual se focaliza un evento anterior a otro posterior al momento de la enunciación; tiene carácter relativo, puesto que no se ordena directamente con relación al momento del habla. Obsérvese el ejemplo siguiente:

(1) Escondednos en la cueva. Y, a la anochecida, llevadnos a vuestro huerto de San Giovanni. Aunque para esa hora ya habremos salido a la luz y estaremos en nuestra casa o en la Señoría [...] (CREA: Fernando Fernán Gómez, 1887: La coartada, Madrid: Antonio Machado).

El evento habremos salido se sitúa con anterioridad al momento temporal indicado por el complemento para esa hora, que, a su vez, es posterior al momento de la enunciación del fragmento concreto. Dado que el evento expresado por el futuro compuesto no se orienta directamente con relación al momento de la enunciación, hablamos de tiempo relativo. En efecto, se orienta como anterior a otro momento a su vez posterior al momento de la enunciación. Esta interpretación se corresponde con la denominación y caracterización del antefuturo por parte de Andrés Bello (1981 [18471-18605]: 405): "Significa que el atributo es anterior a una cosa que respecto del momento en que se habla, es futura”. RAE (2009: \$23,16u) utiliza en estos casos la denominación de "lectura temporal".

2) Interpretación epistémica, en la que se focaliza un evento anterior al momento de la enunciación; tiene carácter absoluto, pues se ordena directamente con relación al momento de la enunciación.

(2) MARUJA- Luisito, buscá a la abuela... jAy! ¿no se habrá caído en una escalera? Y nosotros acá... ¿Cómo la perdimos? (Luis sale). ALBERTO- Y yo qué sé... (CREA: Ana Magnabosco, 1991: Santito mío, Montevideo: Instituto Nacional del Libro).

Se habrá caído expresa un evento anterior al momento de la enunciación o momento del habla, en el que el hablante no se compromete con que, efectivamente, haya ocurrido, sino que lo marca como probable. Para estos casos, RAE (2009: \$23.16u) habla de "lectura modal". Puesto que el evento expresado por el futuro compuesto se orienta directamente con relación al momento de la enunciación o momento del habla, lo consideramos tiempo absoluto. Desde el punto de vista temporal, el futuro compuesto equivale aquí a un pretérito perfecto compuesto. Así afirma al respecto RAE (2009: \$23.16s): "El futuro compuesto de conjetura equivale en unos países a un pretérito compuesto más ese otro componente, como en Me habré equivocado - Probablemente me he equivocado". Pocas líneas después habla de la mayor frecuencia de uso del futuro compuesto con este valor modal: "En diversos recuentos 
estadísticos se ha observado que la interpretación de conjetura (o interpretación modal) del futuro compuesto es mucho más frecuente que la temporal”.

A su vez, como han demostrado Carrasco Gutiérrez (1996 y 1998) y García Fernández (1995 y 2000a), este tiempo es ambiguo desde el punto de vista del aspecto gramatical. Así pues, cada una de las interpretaciones descritas arriba puede recibir una lectura de aoristo o de perfecto. Nos concentraremos en este trabajo en la interpretación de perfecto, que puede manifestar tanto la lectura epistémica como la lectura de anterioridad a un evento orientado al futuro.

Una vez delimitadas las posibles interpretaciones de la forma verbal objeto de estudio, nuestro objetivo es concentrarnos en aquellos casos en que el futuro compuesto aparece con predicados estativos desde el punto de vista del aspecto léxico o modo de acción. Encontramos al respecto afirmaciones como la siguiente de RAE (2009: 1793), en la que se afirma que este tiempo suele aparecer con predicados télicos y no atélicos: "Como el punto intermedio característico de HABRÉ CANTADO localiza un evento, el futuro compuesto es raro con los predicados atélicos que rechazan esta forma de localización”.

Pues bien, en este trabajo demostraremos que son numerosos los casos en que los predicados son atélicos, en particular estativos, que serán objeto de nuestro estudio. Así pues, nuestro interés se centrará en estudiar los casos en que el predicado es de estado y la lectura es de perfecto, ya estemos ante la interpretación temporal, ya ante la modal. Nuestra hipótesis consiste en que la interpretación de perfecto que reciben los predicados estativos corresponde a una de las tres subvariedades que describe Fenn (1987) en su clásico trabajo: la subvariedad de perfecto experiencial. En efecto, cuando aparece un predicado de estado en la lectura de perfecto, la interpretación corresponde a aquella de acuerdo con la cual se focaliza en un determinado momento la experiencia del sujeto como consecuencia de que el evento haya tenido lugar al menos en una ocasión.

Respecto a la procedencia de los ejemplos analizados en nuestro trabajo, la mayoría están tomados del Corpus de referencia del español actual (CREA) de la Real Academia. A estos ejemplos hemos añadido otros seleccionados de corpus disponibles en la red: de la sección de cultura de El Diario.es, de Twitter o de textos literarios, entre otros.

\section{Caracterización del futuro compuesto}

\subsection{La interpretación de anterioridad respecto a un futuro y la interpreta- ción epistémica}

Como hemos señalado arriba, suelen distinguirse dos interpretaciones del futuro compuesto: 1) una interpretación en la que se focaliza un evento anterior a un punto que se orienta con posterioridad al momento de la enunciación o momento del habla; 2) una interpretación de 
acuerdo con la cual el evento se sitúa con anterioridad al momento de la enunciación o momento del habla y expresa modalidad epistémica.

Cada una de estas interpretaciones presenta, a su vez, una ambigüedad desde el punto de vista aspectual, de modo que el resultado son cuatro interpretaciones distintas del futuro compuesto. A estas interpretaciones aspectuales dedicaremos el siguiente apartado.

\subsection{La ambigüedad aspectual del futuro compuesto}

Carrasco Gutiérrez (1996 y 1998: 162-200) y García Fernández (1995 y 2000a) han demostrado que los tiempos compuestos del español son aspectualmente ambiguos entre una interpretación de aoristo y una de perfecto. García Fernández (1995 y 2000a) defiende que las dos interpretaciones aspectuales se corresponden con dos estructuras temporales distintas, lo cual, como señala el estudioso, lo aleja de la idea de Hornstein (1977 y 1990) y de Bertinetto (1986a y 1986b), quienes defienden que las dos interpretaciones se corresponden con la especificación de puntos distintos de la misma línea temporal.

Siguiendo a Klein (1992 y 1994), definimos el aspecto gramatical como la relación entre el tiempo de la situación y el tiempo del foco. El primero lo define el citado estudioso como aquel en que tiene lugar el evento, mientras que el segundo es el tiempo focalizado en cada variedad aspectual. En el perfecto el tiempo del foco es posterior al tiempo de la situación, puesto que no se focaliza el evento, sino la parte posterior a este. Propone Klein distintas representaciones gráficas para las variedades aspectuales. En el caso del perfecto, sería la siguiente:

$-------+++++[+++++]+++++++$

- representa el evento, + la parte anterior y posterior al evento y [ ] la parte del evento focalizada, que en este caso corresponde al resultado.

En el aoristo o perfectivo el tiempo del foco coincide con el tiempo de la situación, puesto que se focaliza el evento completo, tanto el inicio como el final. De acuerdo con las convenciones gráficas de Klein (1992 y 1994), la representación sería la siguiente:

$++++++++++[+--------------+]+++++++++++++$

Entre corchetes se incluye el evento, representado por el signo -, y el inicio y final de este, representado por $+^{2}$.

2 En la definición de aoristo que propone Klein (1992), no se incluye el inicio del evento, por lo que corregimos la definición y la representación gráfica para que corresponda a la focalización del inicio y del final del evento, en lo que seguimos a García Fernández (2000a), quien a su vez sigue a Smith (1991). Véase también al respecto Martínez-Atienza (2012: 41-43). 
Como vamos a mostrar, las dos interpretaciones aspectuales se manifiestan tanto en la lectura del futuro compuesto de anterioridad con respecto a un punto posterior al momento de la enunciación como en la lectura epistémica. Comencemos por la primera:

(3) Si ha reservado y desea cancelar esta ruta, responda al correo que habrá recibido dos horas antes como comprobante de su reserva indicando que desea cancelar su visita. ("Inquisidor. Ruta especial Halloween 2018 en Toledo") https://www.rutasdetoledo.es/tours/ inquisidor-ruta-especial-halloween-2018-en-toledo/(Consultado el 20/11/2018). [AORISTO] ${ }^{3}$

(4) Lo siento, pero soy un poco impaciente, además si no lo hago, cuando lleguemos a tierra, ya se habrá comido a los reenes (“La llegada de Jakes, María, Laila y Yanet” en http://lahistoriadeangela.blogspot.com/2009/04/la-Ilegada-de-jakes-maria-laila-y-yanet.html. Consultado el 20/11/2018). [PERFECTO]

Carrasco (1996: 408 y 409) señala acertadamente que, en la interpretación aspectual de perfecto (como la de (4)), el evento no forma parte de la estructura temporal, por lo que muestra su desacuerdo con Comrie (1981 y 1985), quien habla en estos casos de "vaguedad" para el futuro compuesto. Afirma la estudiosa: "Por consiguiente, no se trataría de que la posición de $\mathrm{E}$ con respecto a $\mathrm{S}$ quede inespecificada sino de que no cuenta para la interpretación del futuro compuesto en ciertos casos" (p. 408)4.

Presentemos, a continuación, los dos ejemplos correspondientes a la lectura epistémica:

(5) Bonito día. Martes y 13. Redondo redondísimo. Festividad de las mártires Felicidad y Lorenza. El sol habrá salido a las 7.30 para ponerse a las 19.19. La luna se acerca a su cuarto menguante en Sagitario y ustedes verán si se casan o se embarcan (CREA: "Se busca público", El Diario Vasco, 13/03/2001). [AORISTO]

(6) PEDRO ¿Las dos y veinte? Marta se habrá dormido ya5. Quedé a las once en subir a dormir con ella (CREA: Juan José Alonso Millán, 1992: Oportunidad: bonito chalet familiar, Madrid: SGAE). [PERFECTO]

Obsérvese que en (5) el complemento temporal a las 7.30 sitúa el evento de la salida del sol, expresado por el futuro compuesto, por lo que corresponde a la interpretación aspectual de aoristo. Como hemos explicado arriba, el evento tiene carácter absoluto y, por tanto, está colocado directamente con anterioridad al momento de la enunciación o momento del habla,

3 Hemos añadido en este ejemplo el complemento temporal dos horas antes para hacer explícita la interpretación de aoristo, de acuerdo con la cual se focaliza el evento completo, no el resultado.

4 De acuerdo con el análisis de Reichenbach (1947) que sigue Carrasco, E representa el evento, y S, el momento del habla o momento de la enunciación.

5 El adverbio ya favorece la interpretación de perfecto (véase García Fernández, 2000a: 216-226). 
esto es, se refiere a las 7.30 del mismo día en que se pronuncia el enunciado. En (6) se focaliza, en el momento de la enunciación, el resultado de un evento que ha tenido lugar antes de dicho momento, estar dormida, como corresponde a la interpretación de perfecto en la que el tiempo del foco es posterior al tiempo de la situación ${ }^{6}$.

\section{Las subvariedades del aspecto perfecto}

Como hemos afirmado arriba, de las dos interpretaciones posibles que manifiesta el futuro perfecto, nos interesa en nuestro trabajo la de perfecto, a la que dedicaremos los tres subapartados siguientes.

Siguiendo los clásicos trabajos de Fenn (1987) y McCoard (1978), dentro del aspecto perfecto distinguimos tres subvariedades: perfecto resultativo, perfecto continuativo o universal y perfecto experiencial, que caracterizaremos e ilustraremos con el futuro compuesto en los siguientes subapartados.

\subsection{El perfecto resultativo}

Esta subvariedad se caracteriza por focalizar los resultados de un evento que ha tenido lugar con anterioridad (véase Altshuler, 2016: 61-82 sobre el propio concepto de resultado). Este significado restringe el tipo de predicados que lo expresan, preferentemente los de carácter télico por estar orientados a un determinado fin o meta intrínseca (entre otros, Bertinetto, 1986b; Mittwoch, 2008: 342; Grønn y Von Stechow, 2021: 4):

(7) En cuanto al poncho, si sigue viviendo en el campo, tal vez lo use en invierno. Para esa época habrán terminado los otoños o los inviernos del patriarca. (CREA: Volodia TeiteIboim, 1988: En el país prohibido. Sin el permiso de Pinochet, Barcelona: Plaza y Janés).

(8) Lo nuevo aquí es el Dino Mountain, una exhibición de bien logrados dinosaurios mecánicos que rugen y escupen, la que podrá visitarse hasta el 6 de septiembre. Para entonces, se supone que ya habrán inaugurado un espacio lleno de feroces felinos [...] (CREA: Revista del Domingo. Domingo en Viaje, 18/07/2004, Santiago de Chile: El Mercurio).

(9) ¿Podrá regresar Mobutu el viernes o para entonces habrá caído Kinshasa?(CREA: ABC Electrónico, 07/05/1997: “Mobutu ‘escapa’ hoy a Gabón, mientras Kabila pide al Ejército zaireño...”).

En el ejemplo (7) encontramos el predicado terminar, en (8) inaugurar y en (9) caer, los tres de carácter télico, de modo que en (7) en el momento temporal indicado por el complemento para esa época se focaliza un resultado, el estado posterior a la finalización de los otoños o de

6 Sobre la sintaxis que corresponde, respectivamente, a la variedad de perfecto y a la de aoristo de las formas compuestas, véase Carrasco (2008: 52-56). 
los inviernos del patriarca. En (8) en el momento indicado por el complemento temporal para entonces, se focaliza el estado posterior a la inauguración de un espacio lleno de feroces felinos y en (9) en el indicado por el complemento para entonces se focaliza el estado posterior a la caída de Kinshasa (véase también Beavers, 2012 sobre esta subvariedad).

\subsection{El perfecto continuativo}

Esta subvariedad se caracteriza por focalizar un evento que ha empezado en un determinado punto, pero del que no se focaliza el final. A menudo la forma verbal aparece combinada con complementos temporales introducidos por la preposición desde, que indican el límite inicial (sobre el perfecto continuativo, véase García Fernández, 2000b):

(10) Contando estas seis semanas que le vienen, Thomas Vermaelen habrá estado 548 días de baja desde que fichó por el Barcelona, en los que se habrá perdido un mínimo de 94 partidos en el Barça, además de 17 más en Roma. Qué calvario (Comentario de Twitter, disponible en https://twitter.com/martinezferran/status/1051799305332379650). (Consultado el 19/11/2018).

(11) ¿Qué no habrá vivido desde entonces? ¿Y quién se sentará esta tarde en su canapé mientras él toque apoyado en el piano? (Arthur Schnitzler, 2010: La señora Berta Garlán, traducción de María Esperanza Romero, Barcelona: Marbot Ediciones, 116).

(12) [...] Para que el visitante, una vez vistos los carruajes, se siga haciendo todas las preguntas que le vengan a la mente sobre este inmenso lugar, y sobre las historias particulares que cada carruaje habrá vivido desde su construcción ("Un museo de carruajes escondido en un vergel sevillano", 30/05/2016. eldiario.es: https://www.eldiario.es/cultura/museo-carruajes-escondido-vergel-sevillano_0_521448379.html).

Obsérvese que en los tres ejemplos anteriores los predicados se combinan con un complemento temporal que indica el límite inicial del evento: desde que fichó por el Barcelona en (10), desde entonces en (11) y desde su construcción en (12). En los tres casos se focaliza un evento que comienza en un momento determinado, pero del que no se focaliza el final en el punto de referencia, lo que corresponde, precisamente, a la subvariedad de perfecto continuativo o universal (véase latridou y otros, 2001 sobre la obligatoriedad del complemento temporal en esta subvariedad).

\subsection{El perfecto experiencial}

Inicia así Fenn (1987: 76) el segundo capítulo de su obra dedicado, precisamente, al denominado Perfect of Experience o Experiential Perfect": "Like the continuative, this kind of perfect also involves an S-inclusive time-span. However, the event is not viewed as occurring through-

7 Según recoge Fenn (1987: 77), McCawley (1971: 104) utiliza un tercer término para hacer referencia 
out the period, as with continuatives, but rather at unspecified points within it" "Como el continuativo, esta clase de perfecto también implica un período que incluye S. Sin embargo, el evento no se presenta como un suceso que ocurre a lo largo del período, como en el continuativo, sino como puntos no especificados dentro de él”).

Efectivamente, como explica el estudioso, podemos afirmar que el perfecto experiencial incluye el momento de la enunciación, pero de un modo diverso respecto al continuativo estudiado arriba, puesto que esta tercera subvariedad no expresa un evento que continúa en el momento de la enunciación o en un punto de referencia distinto, sino que en ese momento lo que se focaliza es la experiencia adquirida por el sujeto como resultado de un evento previo que ha tenido lugar al menos en una ocasión.

Fenn (1987: 77) recoge la explicación de Comrie (1976: 59) sobre el perfecto experiencial, en la que afirma que la distinción entre esta variedad y la de perfecto resultativo está marcada morfológicamente; este es el caso del chino mandarín y el kpelle. El inglés, explica Comrie, no muestra esta distinción morfológicamente (como tampoco muchas otras lenguas europeas), pero se puede manifestar en oposiciones como las de los verbos be y go en contextos como los siguientes:

(13) Bill has been to America (perfecto experiencial).

Bill ha estado en América.

(14) Bill has gone to America (perfecto resultativo).

Bill ha ido a América.

En la segunda, el sujeto está en América en el momento de la enunciación, mientras que en la primera se afirma que el sujeto ha ido a América al menos en alguna ocasión, lo cual no implica que en el momento del habla se encuentre allí. Ilustra con los ejemplos siguientes la diferencia morfológica que manifiesta el chino mandarín: "However, some languages can and do make the distinction regularly. In Mandarin Chinese, the marker of the Experiential Perfect is the toneless suffix-guo. Thus in Chinese we have a contrast between nĭ chĩ-le yúchì-le méiyou, 'have you eaten the shark's fin?' and nĭ chi-gup yúchì méi-you 'have you ever eaten shark's fin? A similar distinction exists in Kpelle" "“Sin embargo, algunas lenguas pueden y estable-

a esta variedad de perfecto, que es el perfecto existencial, y lo ilustra con el ejemplo siguiente: I have read Principia Mathematica 5 times.

He leído Principia Mathematica 5 veces.

latridou y otros (2001) utilizan el mismo término que McCawley al hablar de esta variedad de perfecto.

8 "La lengua kpelle pertenece al grupo mande de la familia congo-kordofán y es hablada en Liberia por un millón de personas" (PROEL).

9 Mittwoch (2008: 324, nota 2), en un trabajo dedicado, precisamente, a las diferencias entre el perfecto resultativo y el experiencial, se refiere a la adquisición de las variedades y señala que el resultativo es adquirido con anterioridad a las otras variedades. 
cen una distinción regularmente. En chino mandarín, el marcador del perfecto experiencial es el sufijo átono -guo. Por lo tanto, en chino tenemos un contraste entre nĬ chĩ -le yúchì -le méi-you, ‘'has comido aleta de tiburón?’ y nĭ chī-gup yúchì méi-you ‘'has comido alguna vez aleta de tiburón? Una distinción similar existe en kpelle”).

También Zandvoort (1957) (apud Fenn, 1987: 76) se ha ocupado del perfecto experiencial y afirma que expresa aquello que ha ocurrido en una o en más de una ocasión en la experiencia del hablante: "expresses what has happened, once or more than once, within the speaker's or writer's experience" ("expresa lo que ha ocurrido una vez o más de una en la experiencia del hablante o del escritor").

Posteriormente, distingue Fenn entre el experiencial general y el limitado, caracterizado el primero de ellos por focalizar la experiencia del sujeto a lo largo de su vida y el segundo en un determinado período de tiempo. Esta distinción no resulta pertinente para el objetivo de nuestro trabajo, por lo que hablaremos exclusivamente de perfecto experiencial.

Ilustramos esta subvariedad de perfecto con algunos ejemplos en futuro compuesto:

(15) Al llegar a U.S.A. pasaréis los tres primeros días alojados en un Hotel, siempre acompañados por un responsable de VIVE que habrá viajado desde Madrid con el grupo, allí nos reuniremos [...] (CREA: Programa impreso, 1994, Programas: espectáculos, universidades...).

(16) En ocasiones, ambas percepciones son muy diferentes entre sí, por eso me atreví a "adivinar" que alguna vez usted habrá experimentado un sobresalto al verse repentinamente en un espejo [...] (CREA: Victor R. Goldsmith, 1993: La salud de tus piernas, Barcelona: Paidós) ${ }^{10,11}$.

Iatridou y otros (2001) explican las diferencias entre el perfecto continuativo y el experiencial con relación a las dos interpretaciones que pueden tener los complementos temporales introducidos por la preposición since en inglés, lo que también puede aplicarse a los introducidos por desde en español. Observemos al respecto los siguientes ejemplos:

10 Nos referimos aquí a la explicación que ofrecen latridou y otros (2001: 192) a propósito de la distinción entre la subvariedad de perfecto resultativo y la de perfecto experiencial. A partir del ejemplo siguiente que proponen: I have lost my glasses, afirman que expresa perfecto resultativo si mientras se pronuncia las gafas siguen perdidas; por el contrario, expresa perfecto experiencial si se pronuncia una vez que se han encontrado.

11 Mittwoch (2008: 326-328) presenta algunas características del contexto que favorecen la interpretación del perfecto experiencial, entre las que figuran la presencia de cuantificadores, como el incluido en este ejemplo en el sintagma nominal alguna vez, que funciona como complemento circunstancial. 
(17) I have been sick since 1990.

He estado enfermo desde 1990.

(Ejemplo de latridou y otros, 2001: 191).

(18) We have lived together since 2014.

Hemos vivido juntos desde 2014.

En ambas oraciones el complemento introducido por since es ambiguo entre una interpretación que las estudiosas denominan "duracional” y una interpretación que llaman "inclusiva”. Desde el punto de vista del aspecto gramatical, cada una de estas lecturas corresponde a una variedad distinta: la duracional corresponde al perfecto continuativo o, de acuerdo con la terminología de las estudiosas, universal, en la que el evento comienza con anterioridad al momento de la enunciación y aún continúa en dicho momento (véase Van der Klis y otros, 2017, y Swart, 2016, para una perspectiva contrastiva sobre el perfecto). La interpretación inclusiva corresponde al perfecto experiencial, de forma que se focaliza en el momento del habla la experiencia del sujeto como resultado de que el evento haya tenido lugar al menos en una ocasión, esto es, en el caso de los ejemplos (17) y (18), que el sujeto haya estado enfermo alguna vez desde 19900 que hayamos vivido juntos en algún período desde 2014. Afirman las estudiosas respecto a esta interpretación para el ejemplo (17): "On the experiential reading, it says that within the interval that extends from 1990 till now there is some (at least one) interval in which I was sick. On this reading, (17) can be continued by I was sick for three months in the fall of 1993"12.

En el siguiente cuadro sintetizamos las tres subvariedades de aspecto perfecto expresadas por el futuro compuesto con algunos de los ejemplos que han aparecido arriba. Recogemos para cada variedad la principal condición que debe cumplirse:

\section{CUADRO 1}

\section{EL FUTURO COMPUESTO Y LAS SUBVARIEDADES DE ASPECTO PERFECTO}

Para entonces, se supone que ya habrán PERFECTO RESULTATIVO

inaugurado un espacio lleno de feroces felinos [...] PRED. TÉLICOS

¿Qué no habrá vivido desde entonces? PERFECTO CONTINUATIVO

COMPLEM. TEMPORAL

[...] me atreví a "adivinar" que alguna vez usted PERFECTO EXPERIENCIAL

habrá experimentado un sobresalto [...] POSIBLE PRESENCIA

CUANTIFICADORES

12 Estamos de acuerdo con la observación de uno de los revisores anónimos de la revista en la que se afirma que la interpretación de perfecto experiencial resulta más natural si aparece un complemento temporal que indica el número de ocasiones en que ha tenido lugar el evento, por ejemplo: "I have been sick two times since 1990". 


\section{El futuro compuesto combinado con predicados estativos}

Hasta aquí hemos estudiado las dos interpretaciones del futuro compuesto: la de anterioridad respecto a un evento futuro y la epistémica. Hemos analizado también las dos lecturas aspectuales que, a su vez, presenta cada una de ellas, a saber, la de aoristo y la de perfecto. Puesto que, de acuerdo con los objetivos de nuestro trabajo, de estas dos interpretaciones nos interesa la de perfecto, a ella hemos dedicado el apartado 3, en particular a caracterizar las tres subvariedades que se distinguen: perfecto resultativo, continuativo y experiencial. A partir de aquí, el objetivo en este cuarto apartado es analizar los casos en que el futuro compuesto aparece con predicados estativos, ya sea en la lectura de anterioridad a un evento futuro, ya en la epistémica, y comprobar a cuál de las tres subvariedades de perfecto corresponde la interpretación.

En RAE (2009: 1792 y 1793) se afirma que el futuro compuesto, en la que denomina "interpretación temporal", aparece en pocas ocasiones con predicados atélicos y, por lo tanto, con estativos:

Como el punto intermedio característico de HABRÉ CANTADO localiza un evento, el futuro compuesto es raro con los predicados atélicos que rechazan esta forma de localización. No se utilizaría, por ejemplo, El año que viene habré sido médico para indicar que el que habla conseguirá su título de Medicina antes de que termine el año entrante. [...] [E]I resultado es irregular en la misma medida en que lo es la variante Este año soy médico, solo admisible en el presente de hechos planificados o previstos [...].

A partir de esta afirmación, demostraremos en nuestro trabajo que no son pocos los ejemplos de futuro compuesto con estados, ejemplos que tomamos de los corpus referidos en el primer apartado del trabajo. Los encontramos tanto en la interpretación de anterioridad con respecto a un evento futuro como en la interpretación de modalidad epistémica. Nos centraremos, pues, en el análisis de estos casos para determinar el valor expresado en ellos ${ }^{13}$.

\subsection{Interpretación de anterioridad respecto a un futuro}

En los siguientes enunciados, encontramos ejemplos de corpus de futuro compuesto con predicados estativos en la interpretación de anterioridad respecto a un futuro:

13 Afirma García Fernández (1995: 384): “Los tiempos perfectos presentan fuertes incompatibilidades con algunas clases de modos de acción verbales (los estativos) y tienden a combinarse preferentemente con otras (los télicos). [...] [E]l aspecto perfecto transforma el modo de acción verbal en estativo, de tal modo que es precisamente incompatible con los verbos estativos". Como ya hemos anunciado, nos concentraremos en el análisis de casos en que el futuro perfecto aparece combinado con predicados estativos. 
(19) No eres mi hermano pero, cuando te maten, habré sido cómplice de tu muerte; habré sido uno de los que te matan. Y no quiero vivir con el peso de mi complicidad (CREA: Luciano G. Egido, 1995: El corazón inmóvil, Barcelona: Tusquets).

(20) Por esto te digo que mucho antes de llegar a Nínive ya habré sido la alegría de la huerta (CREA: Terenci Moix, 2002: El arpista ciego. Una fantasía del reinado de Tutankamón, Barcelona: Planeta).

(21) Abandonar a estas alturas no me parece justo ni con ellos ni con nosotros. Quizás no demos nunca con los que buscamos, pero al menos nosotros habremos sido consecuentes (CREA: José Luis Alegre Cudós, 1989: Locus amoenus, Madrid: Hiperión).

En el primer ejemplo, cuando te maten indica posterioridad respecto al momento de la enunciación y habré sido cómplice y habré sido uno de los que te matan anterioridad respecto a este evento futuro. En (20) el evento futuro respecto al momento de la enunciación está expresado a través del complemento temporal mucho antes de llegar a Nínive, y habré sido la alegría de la huerta es anterior a dicho evento. En (21) el evento expresado por el predicado habremos sido consecuentes es anterior al expresado por Quizás no demos nunca con los que buscamos, que a su vez es posterior al momento de la enunciación. Defendemos en nuestro trabajo que la variedad expresada en estos casos es perfecto y, concretamente, la subvariedad denominada por Fenn (1987) experiencial. En efecto, se focaliza la experiencia del sujeto fruto de un evento previo que ha tenido lugar al menos en una ocasión. En los tres casos se focaliza la parte posterior al final del evento, como corresponde a la interpretación aspectual de perfecto y no de aoristo. En el ejemplo concreto (20), observamos que el futuro compuesto aparece precedido del adverbio ya, que favorece dicha interpretación. Por otro lado, en tanto que predicados atélicos, no podemos tener una interpretación de perfecto resultativo, puesto que esta subvariedad está restringida a los predicados télicos. Por último, lo que distingue principalmente al perfecto continuativo o universal del perfecto experiencial es que en el caso del primero el evento continuaría en el punto de referencia, en este caso en el punto indicado por cuando te maten en (19), por mucho antes de llegar a Nínive en (20) y por no dar nunca con los que buscamos en (21), lo que en estos ejemplos no sucede. Por otro lado, los predicados que reciben esta interpretación aspectual suelen ir acompañados de complementos temporales como los introducidos por desde, que indican el límite inicial (since en el caso del inglés; latridou y otros, 2001), lo que tampoco se produce en estos casos. A partir de este análisis defendemos, pues, que la interpretación de los tres ejemplos corresponde al perfecto experiencial, de forma que en el momento posterior al de la enunciación indicado por las mencionadas secuencias, se focaliza la experiencia del sujeto, que en (19) corresponde a la de haber sido cómplice y haber sido uno de los que te matan, en (20) haber sido la alegría de la huerta y en (21) haber sido consecuentes; en los tres casos, en definitiva, se cumple que el sujeto ha experimentado dichos estados al menos en una ocasión. 


\subsection{Interpretación epistémica}

El siguiente par de enunciados corresponde a la interpretación del futuro compuesto de acuerdo con la cual se focaliza un evento anterior al momento de la enunciación o momento del habla, respecto al cual el hablante no manifiesta su compromiso; es, por tanto, un evento absoluto:

(22) - Pero, ¿tú te has visto cómo vienes? Dónde habrás estado.

- Por ahí escondido -se lamentó, revolviéndose el pelo (CREA: Luis Landero, 1993: Juegos de la edad tardía, Barcelona: Tusquets).

(23) - Supongo que no habré sido yo quien haya intentado influirte -y mosén Alberto esbozó una sonrisa.

- No, no... Creo que lo primero que me influyó fueron los campanarios (CREA: José María Gironella, 1986: Los hombres Iloran solos, Barcelona: Planeta).

(24) ¿Descubriré que el Creador es una memoria sin presente ni futuro? ¿Tú, niño, yo, anciano, habremos sido sólo recuerdos, imágenes insustanciales, sin haber nunca hollado la más mínima realidad? (CREA: Alejandro Jodorowsky, 2001: La danza de la realidad. Chamanismo y psicochamanismo, Madrid: Siruela).

En el ejemplo (22) se interroga en el momento de la enunciación por una posible experiencia del sujeto que corresponde a haber estado en determinado lugar. En (23) se pregunta por la posibilidad por parte del sujeto de haber influido en el interlocutor y en (24) por la de haber sido solo un recuerdo. En los tres ejemplos encontramos predicados estativos, estar y ser, y la interpretación corresponde a la variedad aspectual de perfecto experiencial, explicada arriba. El evento tiene lugar con anterioridad al momento de la enunciación, por lo tanto es absoluto, y se focaliza la experiencia del sujeto fruto de que el evento haya tenido lugar al menos en una ocasión. Como en la interpretación de anterioridad analizada en el apartado precedente, queda excluida la variedad de perfecto resultativo, restringida a los predicados télicos. En efecto, obsérvese que no hay focalización de resultado alguno del evento, como corresponde a la subvariedad de resultativo, pues en los tres casos los predicados son estativos; se focaliza en el momento de la enunciación una experiencia del sujeto derivada de haber estado en algún sitio (22), de que haya sido el hablante quien haya influido en el oyente (23) o de haber sido solo recuerdos (23). Asimismo, queda excluida la interpretación de perfecto continuativo o universal, que focalizaría un evento inconcluso en el momento de la enunciación, pues en estos tres ejemplos los estados han tenido lugar con anterioridad a dicho momento.

Recogemos a continuación en el cuadro 2 algunos de los ejemplos seleccionados anteriormente y su correspondiente interpretación. 


\section{CUADRO 2}

El futuro compuesto con predicados estativos

INTERPRETACIÓN DE ANTERIORIDAD RESPECTO A UN EVENTO POSTERIOR AL ME

[...] pero al menos nosotros

habremos sido consecuentes.

\section{INTERPRETACIÓN EPISTÉMICA}

PERFECTO EXPERIENCIAL

PERFECTO EXPERIENCIAL

\section{Resumen y conclusiones}

El futuro compuesto puede recibir dos interpretaciones: 1) de anterioridad respecto a un evento posterior al momento de la enunciación o momento del habla; 2) de modalidad epistémica. La primera tiene carácter relativo, dado que el evento no se sitúa directamente con relación al momento de la enunciación (a las 20.00 ya habremos llegado), mientras que la segunda tiene carácter absoluto: el evento es anterior al momento de la enunciación (¿Qué habrá pasado?). Cada una de estas interpretaciones, como ha sido demostrado en la bibliografía citada, es aspectualmente ambigua entre un valor de aoristo, de acuerdo con el cual se focaliza el evento completo, y un valor de perfecto, de acuerdo con el cual se focaliza la parte posterior al final del evento. Tenemos, en definitiva, cuatro lecturas distintas del futuro compuesto.

En nuestro trabajo nos hemos concentrado en la interpretación de perfecto que manifiesta esta forma verbal y que, a su vez, presenta tres subvariedades: perfecto resultativo, continuativo o universal y experiencial, diferenciadas entre sí tanto desde el punto de vista semántico como sintáctico. La primera se manifiesta con predicados de carácter télico; la segunda requiere un complemento temporal que implique la continuidad del evento hasta un punto de referencia (complementos introducidos por la preposición desde, complementos con siempre, etc.) y la tercera aparece a menudo acompañada por complementos con cuantificadores numerales o indefinidos como dos veces, en alguna ocasión... Como Fenn (1987) en su trabajo ya clásico demuestra, hay lenguas que manifiestan morfológicamente las diferencias entre el perfecto resultativo y el experiencial, como el chino mandarín o el kpelle.

A menudo se ha hecho referencia en la bibliografía a la restricción que presenta el futuro compuesto por los predicados estativos. Hemos demostrado en este trabajo, sin embargo, que son frecuentes los ejemplos de corpus en los que la variedad de aspecto perfecto expresada por el futuro compuesto se forma con predicados estativos. Como corresponde a la forma verbal objeto de nuestro estudio, las interpretaciones pueden ser bien de anterioridad con respecto a un evento futuro, bien epistémicas. Hemos confirmado nuestra hipótesis de partida, de acuerdo con la cual los ejemplos de perfecto con predicados estativos corresponden a la subvariedad de perfecto experiencial, en la cual se focaliza en el momento de la enunciación la experiencia del sujeto como resultado de que el evento haya tenido lugar al menos 
en una ocasión. Como hemos estudiado, la variedad de perfecto resultativo queda excluida, puesto que corresponde de modo exclusivo a los predicados télicos. Del mismo modo, queda excluida la variedad de perfecto continuativo o universal, que sí puede ser expresada por predicados estativos, pero que, a diferencia del perfecto experiencial, implica que el evento continúa en el momento de la enunciación o en el momento determinado por el contexto sintáctico en el caso del futuro perfecto. En definitiva, hemos demostrado que son numerosos los ejemplos del futuro compuesto con predicados estativos en la interpretación aspectual de perfecto y que corresponden, en particular, a la subvariedad de perfecto experiencial.

\section{Bibliografía citada}

Altshuler, Daniel, 2016: Events, States and Times. An Essay on Narrative Discourse in English, Warsaw/Berlin: De Gruyter Open.

BeAvers, John, 2012: "Resultative Constructions" en Robert I. Binnick (ed.): The Oxford Handbook of Tense and Aspect, Oxford: Oxford University Press, 908-934.

Bello, Andrés, 1981 [1847-18605]: Gramática de la lengua castellana destinada al uso de los americanos, edición de Ramón Trujillo, Santa Cruz de Tenerife: Instituto Universitario de Lingüística Andrés Bello.

Bertinetto, Pier Marco, 1986a: "Intrinsic and Extrinsic Temporal Reference: On Restricting the Notion of 'Reference Time'” en Vincenzo Lo CAScio y Co Vet (eds.): Temporal Structure in Sentence and Discourse, Foris: Dordrecht, 41-78.

Bertinetto, Pier Marco, 1986b: Tempo, Aspetto e Azione nel Verbo Italiano, Florencia: Accademia della Crusca.

Carrasco Gutiérrez, Ángeles, 1996: "La ambigüedad del futuro compuesto” en Carlos Martín Vide (coord.): Lenguajes naturales y lenguajes formales: actas del XII congreso de lenguajes naturales y lenguajes formales, Barcelona: Promociones y Publicaciones Universitarias, 407-414.

Carrasco Gutiérrez, Ángeles, 1998: La correlación de tiempos en español. Tesis doctoral, Universidad Complutense de Madrid.

Carrasco Gutiérrez, Ángeles, 2008: "Los tiempos compuestos del español: formación, interpretación y sintaxis" en Ángeles Carrasco GutiérRez (ed.): Tiempos compuestos y formas verbales complejas, Madrid: Vervuert Iberoamericana, 13-64.

ComRIE, Bernard, 1976: Aspect. An introduction to the study of verbal aspect and related problems, Cambridge / New York: Cambridge University Press. 
Comrie, Bernard, 1981: “On Reichenbach’s Approach to Tense” en Roberta A. Hendrick, Carrie S. Masek y Mary Frances Miller (eds.): Papers from the Seventeenth Regional Meeting of Chicago Linguistic Society, Chicago: University of Chicago, III, 24-30.

Comrie, Bernard, 1985: Tense, Cambridge: Cambridge University Press.

Fenn, Peter, 1987: A Semantic and Pragmatic Examination of the English Perfect, Tübingen: Gunter Narr Verlag.

García Fernández, Luis, 1995: “La interpretación de los tiempos compuestos”, Verba 22, 363-396.

García Fernández, Luis, 2000a: La gramática de los complementos temporales, Madrid: Visor.

García Fernández, Luis, 200ob: “El Perfecto continuativo”, Verba 27, 343-358.

Grønn, Atle, y Arnim von Stechow, 2021: "The Perfect” en Daniel Gutzmann, Lisa Matthewson, Cecile Meier, Hotze Rullmann, Thomas E. Zimmerman (eds.): The Wiley Blackwell Companion to Semantics, New York: Wiley-Blackwell. Cito por la versión disponible en http://folk.uio.no/atleg/perfect_gronn_stechow_oct2017.pdf, fecha de consulta: 28 de noviembre de 2018.

HoRnsteln, Norbert, 1977: “Toward a Theory of Tense”, Linguistic Inquiry 8-3, 521-557.

Hornsteln, Norbert, 1990: As Time Goes by. Tense and Universal Grammar, Cambridge, Massachusetts: The MIT Press.

Iatridou, Sabine, Elena Anagnostopoulou y Roumyana Izvorski, 2001: "Observations about the Form and Meaning of the Perfect" en Michael Kenstowick (ed.): Ken Hale: a Life in Language, Cambridge, Massachusetts: MIT Press, 189-238.

KLeIN, Wolfgang, 1992: “The Present Perfect Puzzle”, Language 68: 3, 525-552.

KLEIN, Wolfgang, 1994: Time in Language, London / New York: Routledge.

Martínez-Atienza, María, 2012: Temporalidad, aspectualidad y modo de acción. La combinación entre formas verbales y complementos temporales en español y su contraste con otras lenguas, Muenchen: Lincom, colección Studies in Romance Linguistics.

McCawley, John, 1971: "Tense and time reference in English" en Charles Fillmore y D. Terence LANGEndoen (eds.): Studies in linguistic semantics, New York: Holt Rinehart, 96-113.

McCoard, Robert W., 1978: The English Perfect: Tense-Choice and Pragmatics Inferences, Amsterdam / New York / Oxford: North-Holland Publishing Company. 
Mıттwoch, Anita, 2008: "The English Resultative perfect and its relationship to the Experiential perfect and the simple past tense", Linguistics and Philosophy 31-3, 323-351.

Promotora Española de Lingǘstica (PROEL) [disponible en http://www.proel.org/index. php?pagina=presentacion, fecha de consulta: 2 de diciembre de 2018].

Real Academia Española, 2009: Nueva gramática de la lengua española, Madrid: Espasa-Calpe.

Real Academia Española: Corpus de referencia del español actual (CREA) [disponible en http:// corpus.rae.es/creanet.html].

Reichenbach, Hans, 1947: Elements of Symbolic Logic, New York: The Free Press.

Sмiтh, Carlota, 1991: The Parameter of Aspect, Dordrecht/Boston/London: Kluwer Academic Publishers.

SWART, Henriëtte de, 2016: "Perfect usage across languages", Questions and answers in Linguistics, de Gruyter Open 3-2, 57-62 [disponible en https://content.sciendo.com/view/journals/ qal/3/2/article-p57.xml, fecha de consulta: 2 de diciembre de 2018].

Van der Klis, Martijn, Bert le Bruyn y Henriëtte de Swart, 2017: "Mapping the PERFECT via Translation Mining" en Proceedings of the 15th Conference of the European Chapter of the Association for Computational Linguistics 2, Short Papers, 497-502.

ZANDvoort, Reinard W., 1957: A Handbook of English Grammar, London: Longmans. 